\title{
CONTROLE POSTURAL E ATIVIDADE FÍSICA EM CRIANÇAS EUTRÓFICAS, COM SOBREPESO E OBESAS
}

\author{
POSTURAL CONTROL AND PHYSICAL ACTIVITY IN EUTROPHIC, OVERWEIGHTAND OBESE CHILDREN
}

CONTROL POSTURAL Y ACTIVIDAD FÍSICA EN NIÑOS CON PESO NORMAL, SOBREPESO Y OBESIDAD

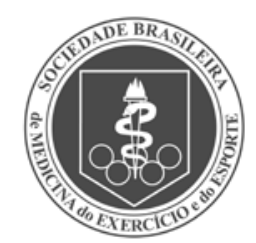

Artigo Original

Original Article Artículo Original
Jessica Caroliny de Jesus Neves ${ }^{1}$ (Fisioterapeuta)

Aryane Karoline Vital de Souza' (Fisioterapeuta)

Dirce Shizuko Fujisawa'

(Fisioterapeuta)

1. Universidade Estadual de Londrina (UEL), Londrina, PR, Brasil.

\section{Correspondência:}

Rua Alagoas, 995, Londrina, PR, Brasil. 86010-520.

jessica_neves_3@hotmail.com

\section{RESUMO}

Introdução: A obesidade infantil vem crescendo nos últimos anos e ocorre, provavelmente, em função da modificação dos hábitos de vida, como sedentarismo e consumo de alimentos inadequados. Na população com excesso de peso, o controle postural, que é um pré-requisito para diversas posturas e atividades, pode ser modificado. Objetivos: Avaliar e comparar o controle postural e a atividade física em crianças eutróficas, com sobrepeso e obesas com oito anos de idade da Rede Municipal de Ensino de Londrina, PR. Além disso, verificar associação entre atividade física e classificação nutricional. Métodos: A amostra foi composta por 346 crianças, divididas em três grupos: eutróficas $(n=205)$, com sobrepeso $(n=69)$ e obesas $(n=72)$, que responderam ao questionário de atividade física para crianças (PAQ-C). A avaliação do controle postural foi realizada na plataforma de força, de preferência, em posição unipodal. Resultados: A prevalência de crianças acima do peso foi de 40,7\% e de sedentárias, 82,9\%, com mediana de três horas diárias em frente à TV. As crianças apresentaram, na posição unipodal, área de COP de 13,41 $\pm 6,77 \mathrm{~cm}^{2}$, o grupo eutrófico apresentou área de COP de 13,97 $\pm 6,67 \mathrm{~cm}^{2}$, o grupo com sobrepeso, $12,34 \pm 5,71 \mathrm{~cm}^{2}$ e os obesos $13,59 \pm 7,94 \mathrm{~cm}^{2}(p=0,785)$. As meninas tiveram melhor desempenho no controle postural do que os meninos ( $p=0,000)$. A frequência $\mathrm{AP}(\mathrm{Hz})$ e a velocidade $\mathrm{ML}(\mathrm{cm} / \mathrm{s})$ apresentaram diferença estatisticamente significante ( $p=0,033$ ep $=0,016$, respectivamente). Também foi identificada associação entre crianças eutróficas e com sobrepeso e atividade física $\left(p=0,013 ; X^{2}=6,186 ;\right.$ Odds Ratio $=0,308 ; p=0,020 ; X^{2}=$ 5,38 , Odds Ratio $=0,293$, respectivamente). Conclusão: Há alta prevalência de excesso de peso e sedentarismo. $O$ excesso de peso não afetou a área de $\mathrm{COP}\left(\mathrm{cm}^{2}\right)$, mas interferiu na frequência AP e na velocidade $\mathrm{ML}$. A atividade física mostrou-se fator protetor em crianças eutróficas e com sobrepeso. Portanto, programas de atividade física devem ser inseridos nas escolas, já que têm caráter preventivo quanto à obesidade e ao sedentarismo.

Descritores: desenvolvimento infantil; equilíbrio postural; atividade motora.

\section{ABSTRACT}

Introduction: Childhood obesity has been growing in recentyears and is probably due to changes in lifestyle, such as inactivity and inadequate food consumption. In the overweight population, postural control, which is a prerequisite for various postures and activities, can be modified. Objectives: To evaluate and compare the postural control and physical activity in eutrophic, overweight and obese children at the age of eight of the public school network of Londrina, PR. In addition, to verify the association between physical activity and nutritional classification. Methods: The sample consisted of 346 children divided into three groups: eutrophic $(n=205)$, overweight $(n=69)$, and obese ( $n=72)$ who answered the questionnaire of physical activity for children (PAQ-C). The assessment of postural control was performed on a force platform, preferably in a unipodal position. Results: The prevalence of overweight children was $40.7 \%$, and the prevalence of sedentary ones was 82.9\%, with a median of three hours daily in front of the TV. The children presented a COP area of $13.41 \pm 6.77 \mathrm{~cm}^{2}$ in the unipodal position, the eutrophic group had COP area of $13.97 \pm 6.67 \mathrm{~cm}^{2}$, the overweight group, $12.34 \pm 5.71 \mathrm{~cm}^{2}$, and obese $13.59 \pm 7.94 \mathrm{~cm}^{2}(p=0.785)$. Girls had better performance in postural control than boys $(p=0.000)$. The AP frequency $(\mathrm{Hz})$ and $\mathrm{ML}(\mathrm{cm} / \mathrm{s})$ presented a statistically significant difference $(p=0.033$ and $p=0.016$, respectively). It was also found association between eutrophic and overweight children and physical activity $\left(p=0.013 ; X^{2}=6.186 ;\right.$ odds ratio $=0.308 ; p=0.020 ; X^{2}=5.38$, odds ratio $=0.293$, respectively). Conclusion: There is a high prevalence of overweight and sedentary children. Overweight did not affect the COP area $\left(\mathrm{cm}^{2}\right)$, but interfered AP frequency and ML velocity. Physical activity proved to be a protective factor in eutrophic and overweight children. Therefore, physical activity intervention programs should be included in schools since they can have a preventive role regarding obesity and sedentary lifestyle.

Keywords: child development; postural balance; motor activity.

\section{RESUMEN}

Introducción: La obesidad infantil ha aumentado en los últimos años, probablemente debido al cambio en el estilo de vida, como la inactividad física y el consumo de alimentos inadecuados. En la población con sobrepeso, el control postural, que es un requisito previo para varias posturas y actividades, puede ser modificado. Objetivos: Evaluar y comparar el control postural y la actividad física en niños con peso normal, sobrepeso y obesos con ocho años de la Red Municipal de Educación de Londrina, PR. Además, verificar la asociación entre actividad física y clasificación nutricional. Métodos: La muestra fue formada por 346 niños, divididos en tres grupos: peso normal ( $n=205$ ), sobrepeso ( $n=69$ ) y obesidad ( $n=72)$, que respondieron el cuestionario de actividad física para niños (PAQ-C). La evaluación 
del control postural se realizó en la plataforma de fuerza, preferiblemente en la posición unipodal. Resultados: La prevalencia de niños con sobrepeso fue del 40,7\%, y el sedentarismo del 82,9\% con una mediana de tres horas al día frente a la televisión. Los niños presentaron, en la posición unipodal, un área de COP de 13,41 $\pm 6,77 \mathrm{~cm}^{2}$, el grupo con peso normal presentó un área de COP de 13,97 $\pm 6,67 \mathrm{~cm}^{2}$, el grupo de sobrepeso $12,34 \pm 5,71 \mathrm{~cm}^{2}$ y los obesos $13,59 \pm 7,94 \mathrm{~cm}^{2}(p=0,785)$. Las niñas tuvieron un mejor control postural que los niños $(p=0,000)$. La frecuencia AP $(\mathrm{Hz})$ y la velocidad $M L(\mathrm{~cm} / \mathrm{s})$ mostraron una diferencia estadisticamente significativa $(p=0,033$ y $p=0,016$, respectivamente). También se encontró asociación entre los niños con peso normal y sobrepeso y la actividad física ( $p=$ 0,$013 ; X^{2}=6,186 ;$ Odds Ratio $=0,308 ; p=0,020 ; X^{2}=5,38$, Odds Ratio $=0,293$, respectivamente). Conclusión: Existe alta prevalencia de sobrepeso e inactividad física. El exceso de peso no afectó el área de $\operatorname{COP}\left(\mathrm{cm}^{2}\right)$, pero interfiere en la frecuencia AP y en la velocidad ML. La actividad física ha demostrado ser un factor de protección para niños con peso normal y sobrepeso. Por lo tanto, deben ser implementados programas de actividad física en las escuelas, ya que tienen un efecto preventivo con respecto a la obesidad y al sedentarismo.

Descriptores: desarrollo infantil; balance postural; actividad motora.

\section{INTRODUÇÃO}

A preocupação com a obesidade e suas consequências em crianças se faz presente nos dias atuais. A Organização Mundial de Saúde (OMS) relata que a obesidade infantil tem crescido em torno de 10 a 40\% na maioria dos países europeus, nos últimos 10 anos, e se tornou epidemia global'.

Estudos relatam que a obesidade na infância e adolescência tende a continuar na fase adulta, se não for convenientemente controlada, levará ao aumento da morbimortalidade e dimuição da expectativa de vida ${ }^{2}$. A etiologia da obesidade é multifatorial, resultando da interação de genes, ambiente, estilos de vida e fatores emocionais ${ }^{3}$. O excesso de peso em crianças e adolescentes ocorre, provavelmente, em função da modificação nos hábitos de vida, como sedentarismo e o consumo de alimentos inadequados.

A atividade física é definida como qualquer movimento do corpo produzido por meio da contração da musculatura esquelética ${ }^{4}$, que gera gasto energético acima do índice de repouso 5 . De maneira geral, existem diferenças quanto às vivências corporais entre crianças ativas e não ativas fisicamente, já que o exercício pode ser considerado como treinamento das instabilidades, pois estão sendo mais vezes desafiadas a manterem o seu equilíbrio e, assim, realizam treinamento natural e contínuo. A literatura tem mostrado que a prática de atividade física regular incorpora e automatiza algumas habilidades, devido à estimulação da estrutura neuromuscular, essencial ao controle postural, pois o exercício é essencial para desenvolver, manter ou recuperar alterações do controle postural ${ }^{6}$.

Portanto, pretende-se estudar o controle postural e atividade física de crianças eutróficas, sobrepesas e obesas, uma vez que a estabilidade pode estar alterada em função de fatores antropométricos, biomecânicos e de vivências corporais. A hipótese é de que crianças eutróficas e ativas apresentem melhor controle postural. Justifica-se também a realização do estudo, devido à escassa publicação sobre os efeitos da obesidade no controle postural em crianças. Destaca-se que, em crianças o controle postural tem especial importância, visto que é fundamental para a aquisição da habilidade motora e o déficit pode provocar alterações posturais. Assim, o objetivo do estudo foi avaliar e comparar o controle postural e atividade física em crianças eutróficas, sobrepesas e obesas com oito anos de idade da Rede Municipal de Londrina- PR. Além disso verificar associação entre atividade física e classificação nutricional.

\section{MATERIAIS E MÉTODOS}

Estudo transversal, com amostra de 346 crianças de oito anos de idade, ambos os sexos, das escolas da Rede Municipal de Londrina, Paraná, Brasil. A idade de oito anos foi estabelecida em função da maturação do controle postural ${ }^{3}$. Os participantes foram selecionados considerando o total de 4.880 crianças matriculadas em 2013, no segundo ano do Ensino Fundamental I da Rede Municipal. O cálculo do tamanho da amostra foi realizado com a fórmula para população finita, com base nos parâmetros: erro amostral de 5\% e intervalo de confiança de 95\%, proporção de $24 \%$, relativo à relação de sobrepeso/obesidade na cidade de Maringá-PR 7 . O tamanho da amostra representativa foi de 266 crianças, já corrigido para população finita, foram recrutadas 20\% a mais considerando possíveis perdas.

As escolas selecionadas eram de todos os quadrantes do município, foi utilizada amostragem estratificada. OTermo de Consentimento Livre e Esclarecido (TCLE) foi encaminhado para assinatura dos pais ou responsáveis dos escolares, no caso de concordância, também o Questionário de Atividade Física para criança/PAQ-C. O projeto de pesquisa foi aprovado pelo Comitê de Ética HU/UEL - Parecer N. 761.965. Para critérios de exclusão foram consideradas crianças com dados incompletos, crianças com impossibilidade de manter a posição ortostática, com alterações ortopédicas, neurológicas e reumáticas, com déficits sensoriais e/ou cognitivo, histórico de doenças neuromusculares ou cirurgias traumato-ortopédicas prévias, doenças agudas ou crônicas e malformações congênitas, queixas de tonturas ou vertigem, déficit visual, e as que fizessem uso de medicação contínua e desnutridas (Figura 1). Avaliação antropométrica: Foram mensurados altura, peso e tamanho dos pés de cada criança. A classificação nutricional realizada por meio do software Anthro WHO Plus, que considera peso, altura, idade e sexo e fornece os valores de escore $Z$. Valores do escore $Z$ menor que - 2 significam baixo peso, entre -2 e +1 eutrofia, entre +1 e +2 sobrepeso e maiores que +2 obesidade. A partir dessa classificação, foram constituídos três grupos de participantes: Eutrófico, Sobrepeso e Obeso ${ }^{8}$. Em seguida foi aplicado o International Obesity Task Force (IOTF) que fornece curvas de IMC (2-18 anos) a partir de dados obtidos de pesquisas populacionais de seis países, incluindo Brasil ${ }^{9}$. A criança classificada como desnutrida pelos dois programas de classificação nutricional foi excluída.

Avaliação do controle postural: Utilizou-se a plataforma de força (PF), considerado padrão-ouro ${ }^{10}$. A PF mede o componente vertical da força de reação do solo, a partir dessa força o software EMG System do Brasil ${ }^{\circledR}$ realiza o cálculo do centro de pressão (COP) e as variáveis referentes à sua variação espaço-temporal. A PF transforma as oscilações corporais (deslocamento do centro de gravidade) em sinais elétricos, que podem ser amplificados, gravados e analisados, visto que seus resultados implicam em medidas de oscilações como área do centro de pressão (Área de COP) $\left(\mathrm{cm}^{2}\right)$, Amplitude ântero-posterior e médio-lateral (Ampl. AP/ Ampl ML) (cm), Velocidade ântero-posterior e médio-lateral (Veloc. AP/Veloc. ML) $(\mathrm{cm} / \mathrm{s})$, Frequência ântero-posterior e médio-lateral (Freq. AP/ Freq. ML) (Hz). 


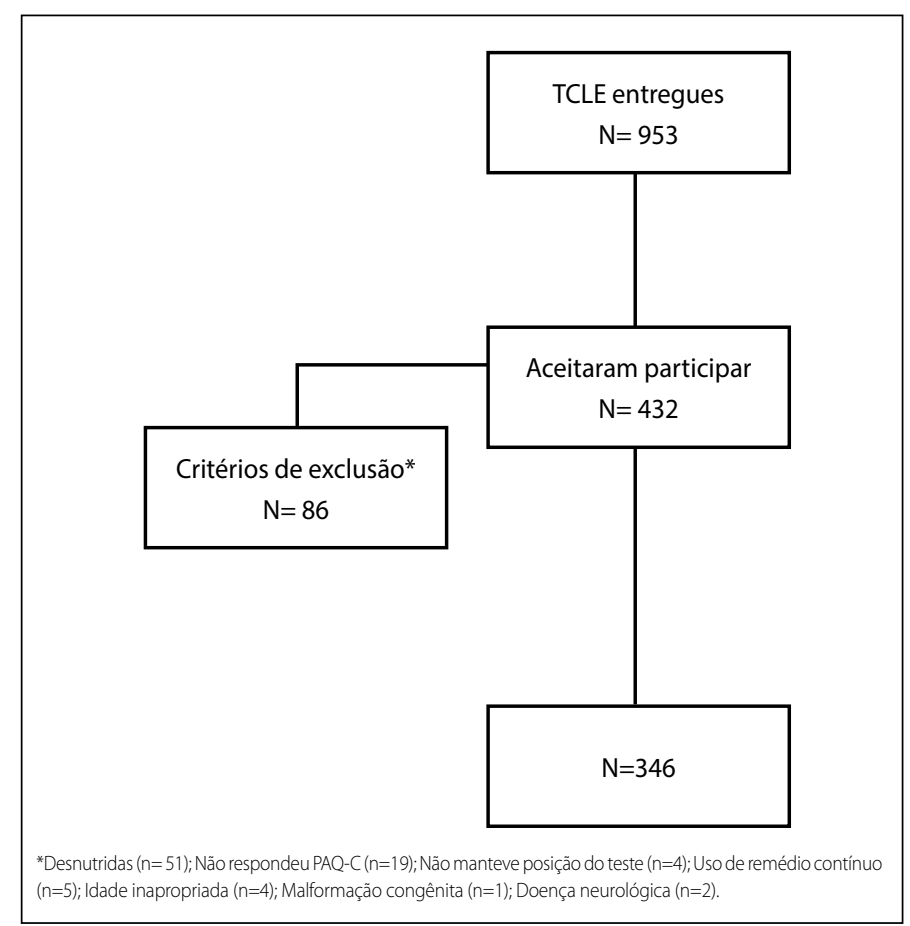

Figura 1. Seleção da amostra.

Avaliação da atividade física em crianças PAQ-C: Composto de 13 questões sobre a prática de esportes e jogos, as atividades físicas na escola e no tempo de lazer, incluindo o final de semana nos últimos sete dias. Cada item do questionário tem valor de um a cinco e o escore final é obtido pela média das questões, representando o intervalo de muito sedentário (1) a muito ativo (5). Os escores dois, três e quatro indicam sedentário, moderadamente ativo e ativo, respectivamente. Na questão número um, que é composta por uma lista de atividades, faz-se necessário transformar a pontuação na escala anteriormente citada, pela divisão do total de pontos na questão pelo número de atividades na lista, incluindo-se também as atividades que tenham sido acrescentadas na seção "outras". O mesmo procedimento foi necessário para a questão 13, que aponta o grau de atividade física em cada dia da semana, o total de pontos é dividido por sete. O escore final foi obtido pela média das questões um a sete, nove e 13. O conjunto de variáveis (idade, sexo e prática de esportes de competição, atividades sedentárias- tempo naTV e computador e/ou videogame e grau de atividade física) foi considerado de forma independente ${ }^{11}$.

\section{Protocolo de avaliação}

A massa corporal foi obtida por meio da pesagem na balança da marca Marte, (modelo LC 200, ano 2010). As crianças foram pesadas descalças, em ortostatismo. A estatura foi mensurada utilizando fita métrica de 150 centímetros (cm), com marcação de 0,1 cm. As crianças permaneciam eretas, pés juntos, braços ao longo do corpo e com os tornozelos em contato com a parede. O comprimento dos pés foi medida por meio de fita métrica, posicionado do calcâneo até o final do primeiro artelho. Para avaliação do controle postural o estudante foi orientado a permanecer em ortostatismo descalças sobre a PF (BIOMEC 410, EMG system), olhando para a marcação à frente, distante a dois metros, dispostos na altura dos olhos, com o tronco em posição ereta com os membros superiores ao lado do corpo, em sala silenciosa e reservada. A posição foi apoio unipodal, com a perna de preferência, por 30 segundos, o membro inferior contralateral permanecia com o quadril em posição neutra e o joelho flexionado a $90^{\circ}$, em três tentativas, com intervalo de descanso de um minuto. O teste foi realizado em apoio unipodal, por ser a posição com maior dificuldade, portanto, a mais discriminativa ${ }^{12}$. Foi adotado frequência de $100 \mathrm{~Hz}$.

\section{Análise estatística}

Os dados coletados foram inseridos no programa Microsoft Excel e analisados no SPSS (versão 2.0). O teste Shapiro-Wilk foi usado para verificar se os dados numéricos apresentavam distribuição normal. Para a análise comparativa do controle postural intergrupos foi utilizado o teste Kruskal-Wallis, o teste Mann Whitney para localizar as diferenças intragrupos em cada variável. Para análise de associação de atividade física com escore Z foi utilizado o teste Qui-quadrado $\left(X^{2}\right)$. Para análise de correlação das medidas antropométricas e controle postural foi utilizado o coeficiente de correlação de Spearman. A significância adotada foi de $p<0,05$. Os resultados foram apresentados em medianas e quartis (25\% e $75 \%$ ).

\section{RESULTADOS}

Os resultados referentes à amostra estão descritas na Tabela 1. Os dados sobre a caracterização da amostra estão descritos na Tabela 2.

As crianças na faixa etária de oito anos apresentaram na posição uni-

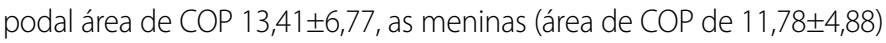
obtiveram melhor controle postural do que o sexo oposto (área de COP de 15,82 $\pm 7,86)$, com diferença estatisticamente significante $(p=0,000)$. A correlação entre medidas antropométricas, como peso, altura com COP não foi identificada ( $p=0,698$ er $=-0,021 ; p=0,837$ er=0,011 respectivamente). A correlação do COP com tamanho dos pés foi fraca ( $p=0,000$ e $r=0,224$,).

Em relação às variáveis da $\mathrm{PF}$, a frequência $\mathrm{AP}(p=0,033)$ e velocidade $\mathrm{ML}$ $(p=0,016)$ apresentaram diferença estatisticamente significante entre crianças eutróficas, sobrepesas e obesas (Tabela 3), não houve diferença nas demais variáveis. Quanto ao PAQ-C e o controle postural, observou-se diferença estatisticamente significante $(p=0,041)$ na amplitude $M L$ em relação a ser ativo ou sedentário (Tabela 4), não houve diferença nas demais variáveis.

Os resultados demonstraram, por meio do teste de Mann Whitney, diferença na área de COP de crianças eutróficas ativas e sedentárias $(p=0,033)$, as ativas tiveram área de COP maior. Por outro lado, as crianças sobrepesas e obesas não apresentaram diferença na área de COP em relação a ser ativa ou sedentária (Tabela 5). Nas questões qualitativas do PAQ-C, as crianças têm mediana de três horas diárias assistindo TV.

Tabela 1. Distribuição quanto ao sexo, preferência da perna utilizada na PF, classificação nutricional e da atividade física.

\begin{tabular}{c|c|c}
\hline \multirow{2}{*}{} & \multicolumn{2}{|c}{ Sexo } \\
\cline { 2 - 3 } & $\mathbf{n}$ & $\%$ \\
\hline Feminino & 180 & 52 \\
\hline Masculino & \multicolumn{2}{|c}{ Preferência Unipodal } \\
\hline \multirow{2}{*}{ Direito } & $\mathbf{n}$ & 48 \\
\hline Esquerdo & 224 & 64,7 \\
\hline \multirow{2}{*}{ Eutrófico } & 122 & 35,3 \\
\hline Sobrepeso & $\mathbf{n}$ & Escore Z \\
\hline Obeso & 205 & 59,3 \\
\hline & 69 & 19,9 \\
\hline & 72 & 20,8 \\
\hline Ativo & $\mathbf{n}$ & PAQ-C \\
\hline Sedentário & 59 & 17,1 \\
\hline
\end{tabular}

Tabela 2. Dados antropométricos e área do COP.

\begin{tabular}{c|c|c|c|c}
\hline Percentil & Massa (Kg) & Estatura $(\mathbf{m})$ & $\begin{array}{c}\text { Comprimento } \\
\text { dos pés }(\mathbf{c m})\end{array}$ & $\begin{array}{c}\text { Área COP } \\
\mathbf{( \mathbf { c m } 2 )}\end{array}$ \\
\hline $25 \%$ & 26,87 & 1,29 & 0,21 & 9,96 \\
\hline Mediana & 30,20 & 1,33 & 0,215 & 13,41 \\
\hline $75 \%$ & 36,30 & 1,37 & 0,225 & 18,47 \\
\hline
\end{tabular}


Tabela 3. Variáveis da PF e classificação nutricional

\begin{tabular}{|c|c|c|c|c|c|c|c|c|c|}
\hline \multirow{3}{*}{ Scor Z } & \multicolumn{3}{|c|}{$\begin{array}{c}\text { Área COP } \\
\left(\mathrm{cm}^{2)}\right.\end{array}$} & \multicolumn{3}{|c|}{$\begin{array}{c}\text { Frequência AP } \\
(\mathrm{Hz})\end{array}$} & \multicolumn{3}{|c|}{$\begin{array}{l}\text { Velocidade ML } \\
(\mathrm{cm} / \mathrm{s})\end{array}$} \\
\hline & \multicolumn{3}{|c|}{ Percentil } & \multicolumn{3}{|c|}{ Percentil } & \multicolumn{3}{|c|}{ Percentil } \\
\hline & $25 \%$ & Mediana & $75 \%$ & $25 \%$ & Mediana & $75 \%$ & $25 \%$ & Mediana & $75 \%$ \\
\hline Eutrófico & 9,49 & 13,97 & 18,95 & 0,64 & $0,75^{*}$ & 0,94 & 3,78 & 4,39". & 5,02 \\
\hline Sobrepeso & 10,29 & 12,34 & 17,49 & 0,59 & $0,73^{\circ}$ & 0,89 & 3,36 & $4,03^{\prime \prime}$ & 4,78 \\
\hline Obeso & 10,52 & 13,59 & 18,91 & 0,66 & $0,84^{* 0}$ & 0,98 & 3,42 & 4,02 . & 4,79 \\
\hline$p$ & \multicolumn{3}{|c|}{0,785} & \multicolumn{3}{|c|}{0,033} & \multicolumn{3}{|c|}{0,016} \\
\hline
\end{tabular}

${ }^{*} p=0,028$ (Eutrófico/Obeso). ${ }^{\circ} p=0,014$ (Sobrepeso/ Obeso). $" \mathrm{p}=0,034$ (Eutrófico/Sobrepeso). $p=0,016$ (Eutrófico/ Obeso).

Tabela 4. Variáveis da plataforma de força e atividade física.

\begin{tabular}{c|c|c|c|c|cc}
\hline \multirow{2}{*}{ PAQ-C } & \multicolumn{3}{c|}{ Área COP $\left(\mathrm{cm}^{2}\right)$} & \multicolumn{3}{c}{ Amplitude ML (cm) } \\
\hline & & Percentil & & & \multicolumn{2}{c}{ Percentil } \\
\hline & $\mathbf{2 5 \%}$ & Mediana & $\mathbf{7 5 \%}$ & $\mathbf{2 5 \%}$ & Mediana & $\mathbf{7 5 \%}$ \\
\hline Ativo & 10,90 & 15,29 & 20,20 & 3,86 & 4,36 & 5,22 \\
\hline Sedentário & 9,72 & 13,16 & 17,70 & 3,63 & 4,18 & 4,80 \\
\hline$p$ & & 0,099 & & & 0,041 & \\
\hline
\end{tabular}

Tabela 5. Variáveis da plataforma de força, classificação nutricional e atividade física.

\begin{tabular}{|c|c|c|c|c|c|c|}
\hline & & PAQ-C & \multicolumn{3}{|c|}{$\begin{array}{c}\text { Área cop } \\
\left(\mathrm{cm}^{2}\right)\end{array}$} & $p$ \\
\hline & & & \multicolumn{3}{|c|}{ Percentil } & \\
\hline & & & $25 \%$ & Mediana & $75 \%$ & \\
\hline \multirow{6}{*}{ EScorE Z } & \multirow{2}{*}{$\begin{array}{l}\text { Eutrófico } \\
(\mathrm{N}=205)\end{array}$} & $\begin{array}{c}\text { Ativo } \\
(\mathrm{N}=40)\end{array}$ & 9,12 & 16,09 & 17,85 & \multirow{2}{*}{0,033} \\
\hline & & $\begin{array}{c}\text { Sedentário } \\
(\mathrm{N}=165)\end{array}$ & 11,08 & 13,21 & 21,23 & \\
\hline & \multirow{2}{*}{$\begin{array}{l}\text { Sobrepeso } \\
\qquad(\mathrm{N}=69)\end{array}$} & $\begin{array}{c}\text { Ativo } \\
(\mathrm{N}=14)\end{array}$ & 10,29 & 12,15 & 17,24 & \multirow{2}{*}{0,887} \\
\hline & & $\begin{array}{c}\text { Sedentário } \\
(\mathrm{N}=55)\end{array}$ & 9,25 & 12,35 & 18,68 & \\
\hline & \multirow{2}{*}{$\begin{array}{l}\text { Obeso } \\
(\mathrm{N}=72)\end{array}$} & $\begin{array}{l}\text { Ativo } \\
(\mathrm{N}=5)\end{array}$ & 10,49 & 12,02 & 19,17 & \multirow{2}{*}{0,898} \\
\hline & & $\begin{array}{c}\text { Sedentário } \\
\qquad(\mathrm{N}=67)\end{array}$ & 10,51 & 13,87 & 25,42 & \\
\hline
\end{tabular}

A análise da classificação de atividade física (sedentário e ativo) por meio do PAQ-C em associação com a classificação nutricional, utilizando o escore $Z$, não ocorreu significância estatística entre crianças eutróficas e sobrepesas $(p=0,888)$. No entanto, entre crianças eutróficas e obesas encontrou-se associação estatisticamente significante entre crianças eutróficas e atividade física $(p=0,013$; $X^{2}=6,186$; Odds Ratio=0,308). Também foi identificada a associação estatisticamente significante entre crianças sobrepesas e atividade física, quando analisado sobrepesos e obesos versus ser ativo ou sedentário $\left(p=0,020 ; X^{2}=5,38 ;\right.$ Odds Ratio $\left.=0,293\right)$.

\section{DISCUSSÃO}

Em nosso estudo, alta prevalência foi encontrada de crianças acima do peso (40,7\%) e sedentárias (82,9\%). Em Londrina a prevalência de excesso de peso registrada é de 18,2\% na faixa etária de 10 a 16 anos $^{13}$. A obesidade está associada com o aumento do sedentarismo em crianças e adolescentes, reflexo do comportamento infantil moderno ${ }^{14}$. A atividade física tem ação protetora sobre o estado nutricional da criança.

Em relação à atividade física, não é de hoje que as crianças encontram-se sedentárias e os meninos são mais ativos. Em Maceió/AL ${ }^{15}$, a prevalência de sedentarismo foi 93,5\% em crianças e adolescentes, sendo maior no sexo feminino. Outro estudo ${ }^{16}, 85 \%$ dos meninos e 94\% das meninas foram classificadas como sedentárias. A tendência das crianças ao sedentarismo se deve ao fato da facilidade ao acesso a televisão, computadores e videogames, reflexo do comportamento infantil moderno, que as predispõem a obesidade ${ }^{17}$.

A mediana da área de COP foi de $13,41 \pm 6,77$, que está entre os relatos

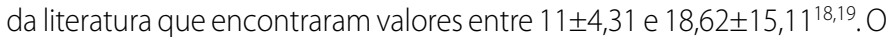
primeiro estudo avaliou 17 crianças com oito e nove anos e o segundo realizado com 40 escolares, incluiu a faixa etária de sete e oito anos, sendo que sete é idade de transição do amadurecimento dos sistemas fornecedores de equilíbrio9. Assim, a variação pode ser decorrente do tamanho da amostra que foi maior e a restrição da faixa etária com crianças somente com oito anos.

Em relação ao sexo feminino apresentar menor área de COP, pode ser devido às diferenças entre os mecanismos de amadurecimento, em meninas ocorre melhor integração sensorial e está disponível mais precocemente do que em meninos aos sete e oito anos de idade ${ }^{20,21}$. Além disso, talvez as meninas sejam mais focadas e prestem mais atenção durante a avaliação na PF, interferindo no seu desempenho. As variáveis antropométricas não tiveram correlação com o controle postural.

As crianças obesas obtiveram frequência AP maiores que as sobrepesas e eutróficas, ou seja, apresentaram maior número de oscilações em 30 segundos para manter a posição unipodal. O excesso de massa corpórea adiposa pode ter influenciado de forma negativa na resposta à oscilação, aumentando a quantidade de movimento necessária ${ }^{22}$. Outro aspecto pode ser a distribuição anormal de massa, principalmente na região do abdômen em crianças obesas, que causa, por exemplo, o desequilíbrio anterior, levando a resposta de magnitude aumentada do torque do tornozelo para recuperar o equilíbrio, maior do que no indivíduo magro, que recuperaria rapidamente a estabilidade ${ }^{23}$. Em relação a variável velocidade $\mathrm{ML}$, as crianças com excesso de peso apresentaram valores menores do que eutróficos, acredita-se que as crianças eutróficas são mais ágeis e, assim, controlam o corpo mais rapidamente para a manutenção da postura ${ }^{24}$.

Nas variáveis numéricas entre ser ativo e sedentário com a área de COP não houve diferença estatisticamente significante, mas a amplitude ML em crianças ativas foi maior, talvez pelo fato do processo de maturação. As maturações dos sistemas estão diretamente ligadas à idade, assim, o melhor controle postural ocorre quando os sistemas estão mais aprimorados ${ }^{25}$. Acredita-se que crianças ativas fisicamente já estejam desenvolvendo a amplitude ML por terem mais experiências motoras. Algumas estratégias de movimento articulares são utilizadas para controlar a posição do corpo no espaço, como as articulações do tornozelo e quadril. Então, uma explicação plausivel para as diferenças com maior instabilidade na posição unipodal seria que quando o indivíduo assume posição mais desafiadora para manutenção do equilíbrio, o quadril produz movimentos mais amplos e rápidos, para controlar e restaurar o equilíbrio em resposta ao forte deslocamento do (CG) ${ }^{24}$.

As crianças eutróficas ativas apresentaram área de COP maior que as sedentárias, diferindo do esperado, visto que o sedentarismo provoca maior inexperiência motora, restringindo a gama de movimentos e podendo provocar menor habilidade dos padrões fundamentais de movimento, entre eles a estabilidade postural ${ }^{3}$. Nesse sentido, sugere-se novos estudos que utilizem ferramentas objetivas para avaliação da atividade física em criança.

Dentre as limitações do estudo está à utilização do PAQ-C, uma vez que é um instrumento subjetivo dependente de memória e foi respondido pelos pais. 


\section{CONCLUSÃO}

Os resultados do presente estudo demonstraram alta prevalência de crianças acima do peso $(40,7 \%)$ e sedentárias (82,9\%) dos escolares da Rede Municipal de Londrina-PR. A obesidade e sobrepeso não afetaram a área de $\operatorname{COP}\left(\mathrm{cm}^{2}\right)$ na população avaliada, visto que não houve diferença estatisticamente significante. Ainda, em relação ao controle postural, os resultados mostraram que o mecanismo é diferenciado quanto à frequência AP (eutróficos e obesos; sobrepesos e obesos) e velocidade ML (eutróficos e sobrepesos; eutróficos e obesos). A atividade física mostrou-se fator protetor em crianças eutróficas e sobrepesas, ou seja, quem realiza atividade física tem menos chance de se tornar obeso e sedentário. Portanto, programas de intervenção de atividades física são recomendados nas escolas, já que possuem caráter preventivo quanto à obesidade e o sedentarismo. Nas crianças obesas também são indicados acompanhamento nutricional. Ainda, outros estudos com delineamento longitudinal devem ser conduzidos.

\section{AGRADECIMENTOS}

A Capes pela aquisição da plataforma de força e a Bolsa - Programa de Demanda Social (DS), a Secretaria Municipal de Educação de Londrina-PR, aos diretores e professores das escolas municipais, aos pais e alunos.

Todos os autores declararam não haver qualquer potencial conflito de interesses referente a este artigo.

CONTRIBUIÇÕES DOS AUTORES: Cada autor contribuiu individual e significativamente para o desenvolvimento do manuscrito. JCJN (0000-0001-8269-5448)* e AKVS $(0000-0003-3705-2755)^{*}$ realizaram a pesquisa bibliográfica e contribuíram com o conceito intelectual do estudo. JCJN e DSF (0000-0001-8427-2860)* foram 0s principais contribuintes na redação do manuscrito e na análise estatística dos dados. JCJN, AKVS e DSF, coletaram e reuniram os dados do estudo e participaram ativamente da discussão dos resultados. DSF realizou a revisão e aprovação final do manuscrito. ${ }^{*} \mathrm{ORCID}$ (Open Researcher and Contributor ID).

\section{REFERÊNCIAS}

1. Organização Mundial da Saúde (OMS). Obesidade: prevenindo e controlando a epidemia global. São Paulo: Roca; 2004 (Série de relatos técnicos da OMS, 894).

2. Fagundes ALN, Ribeiro DC, Naspitz L, Garbelini LEB, Vieira JKP, Silva AP, et al. Prevalência de sobrepeso e obesidade em escolares na região de Parelheiros do município de São Paulo. Rev Paul Pediatr. 2008;26(3):212-7

3. Sociedade Brasileira de Endocrinologia e Metabologia, Sociedade Brasileira de Clínica Médica. Projeto Diretrizes. Obesidade: etiologia [acesso em 2014 ago 03]. São Paulo: AMB e CFM; 2005. Disponível http:// diretrizes.amb.org.br/_BibliotecaAntiga/obesidade-etiologia.pdf.

4. Haskell WL, Kiernan M. Methodologic issues in measuring physical activity and physical fitness when evaluating the role of dietary supplements for physically active people. Am J Clin Nutr. 2000;72(Suppl 2):541S-50.

5. Nahas MV. Atividade física, saúde e qualidade de vida: conceitos e sugestões para um estilo de vida ativo. 2a ed. Londrina: Midiograf; 2001.

6. Gallahue DL, Ozmun JC, Compreendendo o desenvolvimento motor: bebês, crianças, adolescentes e adultos. 3a ed. São Paulo: Phorte; 2005

7. Rosaneli CF, Auler F, Manfrinato CB, Rosaneli CF, Sganzerla C, Bonatto MG, et al. Evaluation of the prevalence and nutritional and social determinants of overweight in a population of schoolchildren: a cross-sectional analysis of 5,037 children. Rev Assoc Med Bras (1992). 2012;58(4):472-6.

8. World Health Organization (WHO). Physical status: the use and interpretation of anthropometry. Geneva:WHO; 1995

9. Cole TJ, Bellizzi MC, Flegal KM, Dietz WH. Establishing a standard definition for child overweight and obesity worldwide: international survey. BMJ. 2000;320(7244):1240-3.

10. Mancini $M$, Horak FB. The relevance of clinical balance assessment tools to differentiate balance deficits. Eur J Phys Rehabil Med. 2010;46(2):239-48.

11. Crocker PR, Bailey DA, Faulkner RA, Kowalski KC, McGrath R. Measurin general levels of physical activity: preliminary evidence for the physical activity questionnaire for older children. Med Sci Sports Exerc. 1997;29(10):1344-9

12. Parreira RB, Boer MC, Rabello L, Costa VS, de Oliveira E Jr, da Silva RA Jr. Age-related differences in center of pressure measures during one-leg stance are time dependent. J Appl Biomech. 2013;29(3):312-6.
13. Christofaro DGD, Andrade SM, Fernandes RA, Ohara D, Dias DF, Freitas Junior IF, et al. Prevalência de fatores de risco para doenças cardiovasculares entre escolares em ondrina-PR: diferenças entre classes econômicas. Rev Bras Epidemiol.2011;14(1):27-35.

14. de Onis M, Blössner M, Borghi E. Global prevalence and trends of overweight and obesity among preschool children. Am J Clin Nutr. 2010;92(5):1257-64.

15. Rivera IR, Silva MAM, Silva RDA,Oliveira BAV, Carvalho ACC. Atividade física, horas de assistência a TV e composição corporal em crianças e adolescentes. Arq Bras Cardiol. 2010;95(2):159-65.

16. da Silva RC, Malina RM. Level of physical activity in adolescents from Niterói, Rio de Janeiro, Brazil. Cad Saude Publica. 2000;16(4):1091-7.

17. Damaso AR, Teixeira LR, Curi CMO. Atividades motoras na obesidade. In: Fisberg M. Atualização em obesidade na infância e adolescência. São Paulo: Atheneu; 2005. p. 131-9.

18. Moraes AG, David AC, Castro OG, Marques BL, Carolino MS, Maia EM. Comparação do equilíbrio postural unipodal entre crianças e adultos. Rev Bras Educ Fís Esporte. 2014; 28(4):571-77.

19. Castro OG, David AG. Deslocamento do centro de pressão nas posições bipodal e unipodal em crianças [dissertação]. Brasília: Faculdade de Educação Física da Faculdade de Brasília; 2013.

20. Geldhof E, Cardon G, De Bourdeaudhuii I, Danneels L, Coorevits P, Vanderstraeten G, et al. Static and dynamic standing balance: test-retest reliability and reference values in 9 to 10 year old children. Eur J Pediatr. 2006;165(11):779-86.

21. Sobera M, Siedlecka B, Syczewska M. Posture control development in children aged 2-7 years old, based on the changes of repeatability of the stability indices. Neurosci Lett. 2011;491(1):13-7.

22. McGraw B, McClenaghan BA, Williams HG, Dickerson J, Ward DS. Gait and postural stability in obese and nonobese prepubertal boys. Arch Phys Med Rehabil. 2000;81(4):484-9.

23. Hue O, Simoneau M, Marcotte J, Berrigan F, Doré J, Marceau P, et al. Body weight is a strong predictor of postural stability. Gait Posture. 2007;26(1):32-8.

24. Duarte M, Freitas SMSF. Revisão sobre posturografia baseada em plataforma de força para avaliação do equilíbrio. Rev Bras Fisioter. 2010;14(3):183-92

25. Steindl R, Kunz K, Schrott-Fischer A, Scholtz AW. Effect of age and sex on maturation of sensory systems and balance control. Dev Med Child Neurol. 2006;48(6):477-82. 